\title{
On backward-time behavior of the solutions to the 2-D space periodic Navier-Stokes equations
}

\section{Sur le comportement rétrograde en temps des solutions périodiques en espace des équations de Navier-Stokes en dimension 2}

\author{
Radu Dascaliuc $^{1}$ \\ Department of Mathematics, Texas A\&M University, College Station, TX 77843, USA
}

Received 25 October 2003; received in revised form 15 July 2004; accepted 27 October 2004

Available online 7 April 2005

\begin{abstract}
In 1995, Constantin, Foias, Kukavica, and Majda had shown that the 2-D space periodic Navier-Stokes equations have a rich set of the solutions that exist for all times $t \in \mathbb{R}$ and grow exponentially in Sobolev $H^{1}$ norm when $t \rightarrow-\infty$. In the present note we show that these solutions grow exponentially (when $t \rightarrow-\infty)$ in any Sobolev $H^{m}$ norm $(m \geqslant 2)$ provided the driving force is bounded in $H^{m-1}$ norm.

(C) 2005 L'Association Publications de l'Institut Henri Poincaré. Published by Elsevier B.V. All rights reserved

\section{Résumé}

En 1995 Constantin, Foias, Kukavica et Majda ont demontré que les équations de Navier-Stokes périodiques dans $\mathbb{R}^{2}$ possèdent un ensemble ample des solutions qui existent pour tout temps $t \in \mathbb{R}$ et qui ont une croissance exponentielle (pour $t \rightarrow-\infty$ ) dans l'espace de Sobolev $H^{1}$. Dans cet article nous montrons que ces solutions ont aussi une croissance exponentielle (pour $t \rightarrow-\infty)$ dans tout espace de Sobolev $H^{m}(m \geqslant 2)$ à condition que la force soit dans l'espace de Sobolev $H^{m-1}$.

(C) 2005 L'Association Publications de l'Institut Henri Poincaré. Published by Elsevier B.V. All rights reserved
\end{abstract}

\footnotetext{
$\overline{1}$ Partially supported by the NSF grant DMS-0139874.

0294-1449/\$ - see front matter (C) 2005 L'Association Publications de l'Institut Henri Poincaré. Published by Elsevier B.V. All rights reserved doi:10.1016/j.anihpc.2004.10.001
} 


\section{Introduction}

One of the remarkable properties of the 2-D space periodic Navier-Stokes equations is the richness of the set of initial data for which the solutions exist for all times $t \in \mathbb{R}$ and increase exponentially as $t \rightarrow-\infty$. These solutions were studied in [3], where, among other results, it was proved that $u(t)$ is such a solution if and only if its Dirichlet quotient $\left|A^{1 / 2} u(t)\right|^{2} /|u(t)|^{2} \rightarrow \lambda_{n}$ as $t \rightarrow-\infty$ (here $|\cdot|$ is the $L^{2}$-norm, $A$ is the Stokes operator, and $\lambda_{n}$ is one of its eigenvalues - see Section 2 for more precise definitions). The invariant set $\mathcal{M}_{n}$ of all the trajectories of these solutions is proved to project entirely onto the spectral space associated with the first $n$ eigenvalues of the Stokes operator (cf. [3]). This fact implied the only known partial answer to the Bardos-Tartar conjecture (cf. [3]). This conjecture (cf. [1]) affirms that the set of initial data for which solutions of the 2-D space periodic Navier-Stokes equations exist for all times is dense in the phase space equipped with the energy norm (a.e. the $L^{2}$-norm in this case). However, in [3] the density was proved in the norm $\left|A^{-1 / 2} \cdot\right|$.

The paper [3] also raised a number of questions regarding the geometric structure of $\mathcal{M}_{n}$. For example, it would be interesting to investigate the relationship between these sets and the other invariant sets of the Navier-Stokes equations, namely the global attractor and inertial manifolds.

Another open question is whether $\bigcup_{n} \mathcal{M}_{n}$ is dense in the energy norm of the phase space, which, if answered affirmatively, would solve the Bardos-Tartar conjecture in the energy norm. The study of higher order quotients on the sets $\mathcal{M}_{n}$ is of particular interest in this respect. In fact, a good result about boundedness of quotients of the form $\left|A^{\alpha} u\right|^{2} /|u|^{\beta}$ would imply the desired density result for $\bigcup_{n} \mathcal{M}_{n}$ via the method presented in [3].

In this paper we prove that the quotients $\left|A^{\alpha} u\right|^{2} /|u|^{4 \alpha}$ are bounded on any $\mathcal{M}_{n}$. (cf. Theorem 2 and its Corollary 1). Our bounds, however, are not sufficient to prove the density of $\bigcup_{n} \mathcal{M}_{n}$ in the energy norm of the phase space. But as a corollary we show that if a solution of the 2-D space periodic Navier-Stokes equation exists for all times and increases exponentially in the energy norm (as $t \rightarrow-\infty)$, than it increases exponentially in any Sobolev norm, provided the driving force is regular (cf. Corollary 2). In particular, the $L^{\infty}$ norm of any derivative of such a solution grows at most exponentially as $t \rightarrow-\infty$.

It is worth mentioning that by a slight modification of the proofs given in this paper one can prove similar results for the 2-D space periodic Navier-Stokes $\alpha$-model and 2-D space periodic Kelvin-filtered Navier-Stokes equations. Note that the analogs of the sets $\mathcal{M}_{n}$ defined for these systems have very similar properties compared to the Navier-Stokes case. In particular for the 2-D space periodic Navier-Stokes $\alpha$-model, $\bigcup_{n} \mathcal{M}_{n}$ is dense in the $L^{2}$ norm, which is still weaker than the energy norm for that system (cf. [10]). On the other hand, for the 2-D space periodic Kelvin-filtered Navier-Stokes equations the density is proved in their energy norm (cf. [11]).

However, not all dissipative systems have the same kind of behavior for negative times. For example, in the case of the 1-D space periodic Kuramoto-Sivashinsky equation it was established that all the solutions outside the global attractor will blow up backward in finite time (cf. [6,7]). The other peculiar example is Burgers' original model for turbulence. Although Burgers' model has a rich set $\mathcal{M}_{1}$, the solutions on it display some surprising dynamical differences from those in the Navier-Stokes case (cf. [4]). Still, it would be interesting to see whether the results similar to the ones presented in this note can also be proved for the set $\mathcal{M}_{1}$ of Burgers' original model for turbulence.

\section{Preliminaries}

We consider the 2-D space periodic Navier-Stokes Equations (NSE) in $\Omega=[0, L]^{2}$ :

$$
\begin{aligned}
& \frac{\mathrm{d}}{\mathrm{d} t} u-v \Delta u+(u \cdot \nabla) u+\nabla p=f, \\
& \nabla \cdot u=0,
\end{aligned}
$$


$u, p \Omega$-periodic, $\int_{\Omega} u=0$,

where $u(t): \mathbb{R}^{2} \rightarrow \mathbb{R}^{2}, p(t): \mathbb{R}^{2} \rightarrow \mathbb{R}$ are unknown functions and $v>0, f \in L^{2}(\Omega)\left(f\right.$ is $\Omega$-periodic, $\left.\int_{\Omega} f=0\right)$ are given.

Let $H$ be the closure in $L^{2}(\Omega)^{2}$ of

$$
\left\{v \in L^{2}(\Omega)^{2}: v \Omega \text {-periodic trigonometric polynomial, } \nabla \cdot v=0, \int_{\Omega} v=0\right\} .
$$

We denote

$$
(v, w):=\int_{\Omega} v \cdot w
$$

and

$$
|v|:=(v, v)^{1 / 2}
$$

the inner product and the norm in $H$.

Let $A=-P_{L} \Delta$ be the Stokes operator (defined on $D(A)=H \cap H^{2}(\Omega)^{2}$ ), where $P_{L}$ is the orthogonal projection from $L^{2}(\Omega)^{2}$ onto $H$. Observe that $A: D(A) \rightarrow H$ is an unbounded positive self-adjoint operator with a compact inverse. Its eigenvalues are $(2 \pi / L)^{2}\left(k_{1}^{2}+k_{2}^{2}\right)$, where $\left(k_{1}, k_{2}\right) \in \mathbb{N}^{2} \backslash\{0,0\}$. We arrange them in the increasing sequence:

$$
(2 \pi / L)^{2}=\lambda_{1}<\lambda_{2}<\cdots .
$$

We will need the following fact about $\left\{\lambda_{n}\right\}$ (cf. [8]).

$$
\limsup _{n \rightarrow \infty}\left(\lambda_{n+1}-\lambda_{n}\right)=\infty
$$

Also, it is obvious that

$$
\lambda_{n+1}-\lambda_{n} \geqslant \lambda_{1}, \quad n \geqslant 1
$$

and

$$
\lim _{n \rightarrow \infty} \lambda_{n}=\infty
$$

Next we denote $\left.B(u, v)=P_{L}((v \cdot \nabla) w)\right)$ and $b(u, v, w)=(B(u, v), w), u, w \in H, v \in D(A)$. Observe that

$$
\begin{aligned}
& b(u, v, w)=-b(u, w, v), \quad u \in H, v, w \in D(A), \\
& b(u, u, A u)=0, \quad u \in D(A) .
\end{aligned}
$$

We will also use the following inequality for $b$ :

$$
|b(u, v, w)| \leqslant c_{0}|u|^{1 / 2}\left|A^{1 / 2} u\right|^{1 / 2}\left|A^{1 / 2} v\right||w|^{1 / 2}\left|A^{1 / 2} w\right|^{1 / 2},
$$

where $u, v, w \in D\left(A^{1 / 2}\right)\left(=H \cap H^{1}(\Omega)^{2}\right)$.

Finally, denote $g=P_{L} f$.

Then the NSE can be written as

$$
\frac{\mathrm{d}}{\mathrm{d} t} u+A u+B(u, u)=g .
$$

We denote by $S(t) u_{0}$ the solution of the NSE which is $u_{0}$ at $t=0$. 
Let

$$
\mathcal{A}=\left\{u_{0} \in \bigcap_{t \geqslant 0} S(t) H: \sup _{t \in \mathbb{R}}\left|S(t) u_{0}\right|<\infty\right\}
$$

be the global attractor of Eq. (2). Refer to [2] or [9] for the comprehensive treatment of Eq. (2).

We will study the $S(t)$-invariant sets

$$
\mathcal{M}_{n}=\mathcal{A} \cup\left\{u_{0} \in \bigcap_{t \geqslant 0} S(t) H: \limsup _{t \rightarrow-\infty} \frac{\left|A^{1 / 2} S(t) u_{0}\right|^{2}}{\left|S(t) u_{0}\right|^{2}} \leqslant \frac{\lambda_{n}+\lambda_{n+1}}{2}:=\bar{\lambda}_{n}\right\} .
$$

We will use the following known facts about $\mathcal{M}_{n}$ (cf. [3]).

Theorem 1. The set $\bigcup_{n} \mathcal{M}_{n}$ is dense in $H$ with the topology of the norm $\left|A^{-1 / 2} \cdot\right|$.

Also:

- If $u(t) \in \mathcal{M}_{n} \backslash \mathcal{M}_{n-1}$ then

$$
\lim _{t \rightarrow-\infty} \frac{\left|A^{1 / 2} u(t)\right|^{2}}{|u(t)|^{2}}=\lambda_{n}
$$

- $u(t) \in \mathcal{M}_{n}$ if and only if

$$
|u(t)|=\mathrm{O}\left(\mathrm{e}^{-v \lambda_{n} t}\right), \quad \text { as } t \rightarrow-\infty ;
$$

- If $u(t) \in \mathcal{M}_{n} \backslash \mathcal{M}_{n-1}$ then

$$
\liminf _{t \rightarrow-\infty} \frac{|u(t)|}{\mathrm{e}^{-v \lambda_{n} t}}>0 .
$$

Moreover, if

$$
\left|u_{0}\right| \geqslant \gamma_{0}:=\max \left\{\frac{2|g|}{v \lambda_{1}}, v\right\}
$$

then

$$
\frac{\left|A^{1 / 2} S(t) u_{0}\right|^{2}}{\left|S(t) u_{0}\right|^{2}} \leqslant \bar{\lambda}_{n}
$$

for all $t \leqslant 0$.

\section{Main result}

For every $\theta \geqslant 0$ and $g \in D\left(A^{\theta}\right)$ define

$$
G_{\theta}=\frac{\left|A^{\theta} g\right|}{v^{2} \lambda_{1}^{\theta+1}},
$$

the generalized Grashoff number.

Our main goal is to prove the following

Theorem 2. Let $\theta=k / 2, k \in \mathbb{N} \backslash\{0\}$, and $g \in D\left(A^{\theta}\right)$. Then for every $u_{0} \in \mathcal{M}_{n}$ such that $\left|u_{0}\right| \geqslant \gamma_{0}$, there exists a positive constant $M_{\theta}\left(G_{\theta}\right)$ depending only $\theta, c_{0}$ (where $c_{0}$ the constant from (1)), and $G_{\theta}$ such that

$$
\frac{\left|A^{\theta} u_{0}\right|^{2}}{\left|u_{0}\right|^{4 \theta}} \leqslant \frac{M_{\theta}\left(G_{\theta}\right)}{v^{4 \theta-2}}{\overline{\lambda_{n}}}^{2 \theta}
$$


Moreover, if $\theta>1$ than there exists a positive constant $N_{\theta}\left(G_{\theta-1 / 2}\right)$, that depends only on $\theta, c_{0}$, and $G_{\theta-1 / 2}$ such that

$$
\int_{-\infty}^{t_{0}} \frac{\left|A^{\theta} u\right|^{2}}{|u|^{4 \theta-2}} \mathrm{~d} \tau<\frac{N_{\theta}\left(G_{\theta-1 / 2}\right)}{v^{4 \theta-3}}{\overline{\lambda_{n}}}^{2 \theta-1},
$$

where $u(t)$ is a solution of the NSE satisfying $u\left(t_{0}\right)=u_{0}$.

Also, if $\theta \geqslant 1 / 2$ then

$$
\lim _{t \rightarrow-\infty} \frac{\left|A^{\theta} u(t)\right|^{2}}{|u(t)|^{4 \theta}}=0 .
$$

Observe that (11) expands the estimate (9) from Theorem 1 to the quotients involving higher powers of the operator $A$. In fact, these estimates hold for any power of the operator.

Corollary 1. Let $\alpha>1 / 2$ and $g \in D\left(A^{\theta}\right)$, where $\theta=([2 \alpha]+1) / 2$. Then for every $u_{0} \in \mathcal{M}_{n}$ with $\left|u_{0}\right|>\gamma_{0}$, there is a constant $M_{\alpha}$ (depending only on $\theta, G_{([2 \alpha]+1) / 2}$, and $\left.c_{0}\right)$ such that

$$
\frac{\left|A^{\alpha} u_{0}\right|^{2}}{\left|u_{0}\right|^{4 \alpha}} \leqslant \frac{M_{\alpha}}{v^{4 \alpha-2}} \bar{\lambda}_{n}^{2 \alpha} .
$$

Proof. Let $\theta=([2 \alpha]+1) / 2$. Observe that $\theta \geqslant \alpha$. Then, by interpolation,

$$
\begin{aligned}
\frac{\left|A^{\alpha} u_{0}\right|^{2}}{\left|u_{0}\right|^{4 \alpha}} & \leqslant\left(\frac{\left|A^{\theta} u_{0}\right|^{2}}{\left|u_{0}\right|^{4 \theta}}\right)^{(2 \alpha-1) /(2 \theta-1)}\left(\frac{\left|A^{1 / 2} u_{0}\right|^{2}}{\left|u_{0}\right|^{2}}\right)^{(2 \theta-2 \alpha) /(2 \theta-1)} \\
& \leqslant\left(\frac{M_{\theta}}{v^{4 \theta-2}}{\overline{\lambda_{n}}}^{2 \theta}\right)^{(2 \alpha-1) /(2 \theta-1)}{\overline{\lambda_{n}}}^{(2 \theta-2 \alpha) /(2 \theta-1)}=\frac{M_{\theta}^{(2 \alpha-1) /(2 \theta-1)}}{v^{4 \alpha-2}}{\overline{\lambda_{n}}}^{2 \alpha},
\end{aligned}
$$

and thus, (14) holds with $M_{\alpha}=M_{\theta}^{(2 \alpha-1) /(2 \theta-1)}$.

Another consequence of Theorem 2 is that on $\mathcal{M}_{n}$ any Sobolev norm of a solution will grow exponentially for negative time.

Corollary 2. Suppose $u(t) \in \mathcal{M}_{n} \backslash \mathcal{A}$ and $g \in D\left(A^{m / 2}\right)$ then

$$
\left|A^{m / 2} u(t)\right|^{2} \leqslant \mathrm{O}\left(\mathrm{e}^{-2 m v \lambda_{n} t}\right), \quad t \rightarrow-\infty .
$$

Moreover, if $m \geqslant 2$, then for any $\alpha=\left(\alpha_{1}, \alpha_{2}\right)$ with $\alpha_{1}, \alpha_{2} \geqslant 0, \alpha_{1}+\alpha_{2} \leqslant m-2$, we have

$$
\left|D^{\alpha} u\right|_{L^{\infty}}=\mathrm{O}\left(\mathrm{e}^{-\left(\alpha_{1}+\alpha_{2}+2\right) \nu \lambda_{n} t}\right), \quad t \rightarrow-\infty,
$$

where

$$
D^{\alpha} u\left(x_{1}, x_{2}\right)=\frac{\partial^{\alpha_{1}+\alpha_{2}} u}{\partial^{\alpha_{1}} x_{1} \partial^{\alpha_{2}} x_{2}} .
$$

In particular, when $g \in C^{\infty}(\Omega)$, any solution $u$ of the NSE which exists for all times and increases exponentially as $t \rightarrow-\infty$ in the phase space $H$, will also increase exponentially as $t \rightarrow-\infty$ in any Sobolev space $H_{\mathrm{per}}^{m}(\Omega)^{2}=$ $W_{\text {per }}^{2, m}(\Omega)^{2}(m \geqslant 0)$. Moreover, the $L^{\infty}$ norm of any (space) derivative of $u$ will also increase exponentially as $t \rightarrow-\infty$. 
Proof. Recall that the Sobolev norm in $H_{\mathrm{per}}^{m}(\Omega)$ is equivalent to the norm

$$
|\cdot|_{m}:=\left(\left|A^{m / 2} \cdot\right|^{2}\right)^{1 / 2} \text {. }
$$

Note that by Theorem $1 u(t) \in \mathcal{M}_{n} \backslash \mathcal{A}$ implies that $|u(t)|_{m}$ grows at least exponentially as $t \rightarrow-\infty$, and $|u(t)|^{2}=$ $\mathrm{O}\left(\mathrm{e}^{-v \lambda_{n} t}\right)$ as $t \rightarrow-\infty$.

On the other hand, according to Theorem 2,

$$
\left|A^{m / 2} u(t)\right|^{2} \leqslant \frac{M_{m / 2}}{v^{2 m-2}} \lambda_{n}^{k}|u|^{2 m}=\mathrm{O}\left(\mathrm{e}^{-2 m v \lambda_{n} t}\right) .
$$

Thus, $u(t)$ increases exponentially in $H_{\text {per }}^{m}(\Omega)^{2}$ as $t \rightarrow-\infty$.

To prove the second part of the corollary we apply the Sobolev Embedding Theorem to obtain that

$$
\left|D^{\alpha} u\right|_{\infty} \leqslant C\left|D^{\alpha} u\right|_{H^{2}(\Omega)},
$$

for any multi-index $\alpha=\left(\alpha_{1}, \alpha_{2}\right) \in \mathbb{N}^{2}$. Here we are writing

$$
D^{\alpha} u\left(x_{1}, x_{2}\right)=\frac{\partial^{\alpha_{1}+\alpha_{2}} u}{\partial^{\alpha_{1}} x_{1} \partial^{\alpha_{2}} x_{2}} .
$$

Observe that by the first part of the corollary, $\left|D^{\alpha} u(t)\right|_{H^{2}(\Omega)}=\mathrm{O}\left(\mathrm{e}^{-\left(\alpha_{1}+\alpha_{2}+2\right) v \lambda_{n} t}\right)$ as $t \rightarrow-\infty$. Consequently, we also have that $\left|D^{\alpha} u\right|_{\infty}=\mathrm{O}\left(\mathrm{e}^{-\left(\alpha_{1}+\alpha_{2}+2\right) \nu \lambda_{n} t}\right)$ as $t \rightarrow-\infty$.

\section{The proof of the main result}

For convenience we will use the following notation:

\section{Notation 1.}

$$
\begin{aligned}
& \lambda:=\frac{\left|A^{1 / 2} u\right|^{2}}{|u|^{2}}, \\
& \mu:=\frac{|A u|^{2}}{|u|^{4}}, \\
& \xi:=(A-\lambda) \frac{u}{|u|}, \\
& \sigma:=\left(A-\frac{3}{2} \lambda\right) \frac{A^{1 / 2} u}{|u|^{2}}, \\
& \bar{\lambda}_{n}:=\frac{\lambda_{n+1}+\lambda_{n}}{2}, \\
& \mu_{\theta, m}:=\frac{\left|A^{\theta} u\right|^{2}}{|u|^{m}} .
\end{aligned}
$$

First, we will prove the following useful lemma.

Lemma 1. Let $u$ be a solution of the NSE that exists for all times and satisfies $\left|u\left(t_{0}\right)\right|>\gamma_{0}$ for some $t_{0}$. Then for any $t \leqslant t_{0}$ and any $m \geqslant 1$,

$$
\frac{2}{3 m} \frac{1}{|u(t)|^{m}} \leqslant v \int_{-\infty}^{t} \frac{\lambda(\tau)}{|u(\tau)|^{m}} \mathrm{~d} \tau \leqslant \frac{2}{m} \frac{1}{|u(t)|^{m}} .
$$


Also, if $u\left(t_{0}\right) \in \mathcal{M}_{n} \backslash \mathcal{A}$, then

$$
v \int_{-\infty}^{t} \lambda(\tau)|\xi(\tau)|^{2} \mathrm{~d} \tau \leqslant \frac{1}{2}\left(\lambda_{n}^{2}-\lambda^{2}(t)\right)+\frac{|g|^{2}}{v^{2}|u(t)|^{2}}
$$

and

$$
v \int_{-\infty}^{t} \mu(\tau) \mathrm{d} \tau \leqslant \mathrm{O}\left(\frac{1}{|u(t)|^{2}}\right), \quad \text { for } t \rightarrow-\infty
$$

Proof. From (2) we obtain

$$
\frac{1}{2} \frac{\mathrm{d}}{\mathrm{d} t}|u|^{2}+v \lambda|u|^{2}=(g, u),
$$

from which we get

$$
\frac{1}{|u|^{m+1}} \frac{1}{2} \frac{\mathrm{d}}{\mathrm{d} t}|u|+v \frac{\lambda}{|u|^{m}}=\left(g, \frac{u}{|u|^{m+2}}\right) .
$$

Thus

$$
v \int_{-\infty}^{t} \frac{\lambda}{|u|^{m}} \mathrm{~d} \tau-\int_{-\infty}^{t}\left(g, \frac{u}{|u|^{m+2}}\right) \mathrm{d} \tau=\frac{1}{m} \frac{1}{|u(t)|^{m}}
$$

Notice that for $t \leqslant t_{0}$

$$
\left|\int_{-\infty}^{t}\left(g, \frac{u}{|u|^{m+2}}\right) \mathrm{d} \tau\right| \leqslant \int_{-\infty}^{t} \frac{g}{|u|^{m+1}} \mathrm{~d} \tau \leqslant \int_{-\infty}^{t}\left(\frac{g}{\nu \lambda_{1}} \frac{1}{|u|}\right) \frac{1}{|u|^{m}} \mathrm{~d} \tau \leqslant \frac{1}{2} \nu \int_{-\infty}^{t} \frac{\lambda_{1}}{|u|^{m}} \mathrm{~d} \tau \leqslant \frac{1}{2} \nu \int_{-\infty}^{t} \frac{\lambda}{|u|^{m}} \mathrm{~d} \tau,
$$

since $|u(t)| \geqslant \gamma_{0}\left(\geqslant \frac{2 g}{v \lambda_{1}}\right)$ and $\lambda(t) \geqslant \lambda_{1}$ for all $t \leqslant t_{0}$. Thus, returning to (19) we get

$$
v \int_{-\infty}^{t} \frac{\lambda}{|u|^{m}} \mathrm{~d} \tau-\frac{1}{2} v \int_{-\infty}^{t} \frac{\lambda}{|u|^{m}} \mathrm{~d} \tau \leqslant \frac{1}{m} \frac{1}{|u(t)|^{m}}
$$

and

$$
v \int_{-\infty}^{t} \frac{\lambda}{|u|^{m}} \mathrm{~d} \tau+\frac{1}{2} \nu \int_{-\infty}^{t} \frac{\lambda}{|u|^{m}} \mathrm{~d} \tau \geqslant \frac{1}{m} \frac{1}{|u(t)|^{m}}
$$

for all $t \leqslant t_{0}$, from which the relation (15) readily follows.

In order to prove (16), we observe that

$$
\frac{1}{2} \frac{\mathrm{d}}{\mathrm{d} t}\left|A^{1 / 2} u\right|^{2}+v|A u|^{2}=(g, A u),
$$

which, together with (18), implies that

$$
\frac{1}{2} \frac{\mathrm{d}}{\mathrm{d} t} \lambda+v|\xi|^{2}=\left(\frac{g}{|u|}, \xi\right),
$$

from which we obtain

$$
\frac{1}{2} \frac{\mathrm{d}}{\mathrm{d} t} \lambda^{2}+v \lambda|\xi|^{2} \leqslant \frac{\lambda|g|^{2}}{v|u|^{2}} .
$$


By integrating the relation above, using (15) for $m=2$ as well as the fact that $\lambda(t) \rightarrow \lambda_{n}$ as $t \rightarrow-\infty$ (cf. results from [3] summarized in Theorem 1), we get

$$
\frac{1}{2}\left(\lambda^{2}(t)-\lambda_{n}^{2}\right)+v \int_{-\infty}^{t} \lambda|\xi|^{2} \mathrm{~d} \tau \leqslant \frac{|g|^{2}}{v^{2}|u(t)|^{2}}
$$

which implies the inequality (16) from the statement of the lemma.

Finally, to prove (17) consider

$$
\frac{1}{2} \frac{\mathrm{d}}{\mathrm{d} t} \frac{\lambda}{|u|^{2}}=\frac{-v|A u|^{2}+(g, A u)}{|u|^{4}}-2 \frac{\lambda}{|u|^{2}} \frac{-v\left|A^{1 / 2} u\right|^{2}+(g, u)}{|u|^{2}},
$$

from where

$$
\frac{1}{2} \frac{\mathrm{d}}{\mathrm{d} t} \frac{\lambda}{|u|^{2}} \leqslant-v \mu+\frac{|g|^{2}}{|u|^{2}} \mu^{1 / 2}+2 v \frac{\lambda^{2}}{|u|^{2}}+2 \frac{\lambda|g|}{|u|^{3}} .
$$

Consequently

$$
\frac{\mathrm{d}}{\mathrm{d} t} \frac{\lambda}{|u|^{2}}+v \mu \leqslant \frac{|g|^{2}}{|u|^{2}}+4 v \frac{\lambda^{2}}{|u|^{2}}+4 \frac{\lambda|g|}{|u|^{3}} .
$$

Thus, by integrating the previous inequality and using (15) we obtain

$$
v \int_{-\infty}^{t} \mu(\tau) \mathrm{d} \tau \leqslant \mathrm{O}\left(\frac{1}{|u(t)|^{2}}\right) \quad \text { for } t \rightarrow-\infty
$$

Let $u(t)$ be a solution of the NSE such that $u(t) \in \mathcal{M}_{n}$. Our first result is

Proposition 1. If $g \in D(A)$ and

$$
|u(0)| \geqslant \gamma_{0},
$$

then for every $t \leqslant 0$ we have

$$
\mu(t)+\mathrm{e}^{-3} v \int_{-\infty}^{t} \lambda(\tau) \mu(\tau) \mathrm{d} \tau \leqslant \frac{\mathrm{e}^{4}}{2 v^{2}}\left(\lambda_{n}^{2}-\lambda^{2}(t)\right)+\frac{K_{1} \lambda_{1}^{2}+(13 / 4) \mathrm{e}^{4} \bar{\lambda}_{n}^{2}}{|u(t)|^{2}},
$$

where $K_{1}=e^{4}\left(c_{0} G_{0}+G_{1}\right)$ with $c_{0}$ - the constant from the inequality (1). Moreover,

$$
\frac{v}{4} \mathrm{e}^{-3} \int_{-\infty}^{t} \frac{\left|A^{3 / 2} u\right|^{2}}{|u|^{4}} \mathrm{~d} \tau \leqslant \frac{\mathrm{e}^{4}}{2 v^{2}}\left(\lambda_{n}^{2}-\lambda^{2}(t)\right)+\frac{K_{1} \lambda_{1}^{2}+(13 / 4) \mathrm{e}^{4} \bar{\lambda}_{n}^{2}}{|u(t)|^{2}}
$$

for any $t \leqslant 0$.

Proof. Observe that since $g \in D(A)$, we have

$$
\frac{1}{2} \frac{\mathrm{d}}{\mathrm{d} t} \mu=\frac{-v\left|A^{3 / 2} u\right|^{2}-b(A u, u, A u)+(A g, A u)}{|u|^{4}}-2 \mu \frac{-v\left|A^{1 / 2} u\right|^{2}+(g, u)}{|u|^{2}},
$$

so,

$$
\frac{1}{2} \frac{\mathrm{d}}{\mathrm{d} t} \mu=-v\left(\mu_{3 / 2,4}-2 \mu \lambda\right)-\frac{b(A u, u, A u)}{|u|^{4}}+\frac{(A g, A u)}{|u|^{4}}-2 \mu \frac{(g, u)}{|u|^{2}} .
$$


Thus,

$$
\begin{aligned}
\frac{1}{2} \frac{\mathrm{d}}{\mathrm{d} t} \mu+v \lambda \mu= & -v|\sigma|^{2}+\frac{9 v}{4} \frac{\lambda^{3}}{|u|^{2}}-\frac{b(A u, u, A u)}{|u|^{4}} \\
& +\left(\frac{A g}{|u|^{2}}, \frac{A u}{|u|^{2}}\right)-2 \mu\left(g, \frac{u}{|u|^{2}}\right) .
\end{aligned}
$$

Note that

and thus

$$
\begin{aligned}
\frac{|b(A u, u, A u)|}{|u|^{4}} & =\frac{b(A u-\lambda u, u, A u-\lambda u)}{|u|^{4}} \leqslant \frac{c_{0}|\xi|\left|A^{1 / 2} \xi\right|\left|A^{1 / 2} u\right|}{|u|^{2}} \\
& =c_{0} \lambda^{1 / 2}|\xi| \frac{\left|A^{1 / 2} \xi\right|}{|u|}=c_{0} \lambda^{1 / 2}|\xi|\left(|\sigma|^{2}+\lambda \mu-\frac{5}{4} \frac{\lambda^{3}}{|u|^{2}}\right)^{1 / 2}
\end{aligned}
$$

$$
\frac{|b(A u, u, A u)|}{|u|^{4}} \leqslant \frac{c_{0}^{2}}{2 v} \lambda|\xi|^{2}+\frac{v}{2}\left(|\sigma|^{2}+\lambda \mu-\frac{5}{4} \frac{\lambda^{3}}{|u|^{2}}\right) .
$$

Now, going back to (21) we get

$$
\frac{1}{2} \frac{\mathrm{d}}{\mathrm{d} t} \mu+v \lambda \mu \leqslant-\frac{v}{2}|\sigma|^{2}+\frac{v}{2} \lambda \mu-\frac{5 v}{8} \frac{\lambda^{3}}{|u|^{2}}+\frac{9 v}{4} \frac{\lambda^{3}}{|u|^{2}}+\frac{c_{0} \lambda}{2 v}|\xi|^{2}+\frac{|A g|}{|u|^{2}} \mu^{1 / 2}-2 \mu\left(g, \frac{u}{|u|^{2}}\right) .
$$

Observe that

\section{Consequently}

$$
\frac{|A g|}{|u|^{2}} \mu^{1 / 2} \leqslant \frac{1}{2 v^{3} \lambda_{1}} \frac{|A g|^{2}}{|u|^{2}}+\frac{v^{3} \lambda_{1}}{2} \frac{\mu}{|u|^{2}} .
$$

$$
\frac{\mathrm{d}}{\mathrm{d} t} \mu+v \lambda \mu \leqslant\left[\frac{v^{3} \lambda_{1}}{|u|^{2}}-4\left(g, \frac{u}{|u|^{2}}\right)\right] \mu-v|\sigma|^{2}+\frac{c_{0} \lambda}{v}|\xi|^{2}+\frac{1}{|u|^{2}}\left(\frac{13 v}{4} \lambda^{3}+\frac{|A g|^{2}}{v^{3} \lambda_{1}}\right) .
$$

Note that the conditions of the proposition imply that $\lambda(t) \leqslant \bar{\lambda}_{n}$, for all $t \leqslant 0$. Let us denote

$$
\begin{aligned}
& \Gamma_{n}:=\frac{13 v}{4} \bar{\lambda}_{n}^{2}+\frac{|A g|^{2}}{v^{3} \lambda_{1}^{2}}, \\
& \beta:=\frac{v^{3} \lambda_{1}}{|u|^{2}}-4\left(g, \frac{u}{|u|^{2}}\right) .
\end{aligned}
$$

Then, by the Gronwall inequality,

$$
\mu(t) \leqslant \mu\left(t_{0}\right) \mathrm{e}^{\int_{t_{0}}^{t} \beta}+\int_{t_{0}}^{t}\left(-v|\sigma|^{2}-v \lambda \mu+\frac{c_{0}}{v} \lambda|\xi|^{2}+\frac{\Gamma_{n} \lambda}{|u|^{2}}\right) \mathrm{e}^{\int_{\tau}^{t} \beta} \mathrm{d} \tau .
$$

Observe that cf. Theorem 1 ,

$$
\liminf _{t \rightarrow-\infty} \frac{|u(t)|^{2}}{\mathrm{e}^{-v \lambda_{1} t}}>0
$$

and so $\beta(\tau)$ is bounded and absolutely integrable on the interval $(-\infty, t]$. Moreover, by Lemma $1,\left(c_{0} / v\right) \lambda|\xi|^{2}+$ $\Gamma_{n} \lambda /|u|^{2}$ is also absolutely integrable on $(-\infty, t]$. On the other hand, from (17) we conclude that there exists a sequence $t_{n}^{0} \rightarrow-\infty$ such that $\mu\left(t_{n}^{0}\right) \rightarrow 0$. Thus, by taking $t_{0}=t_{n}^{0}$ and letting $n \rightarrow \infty$, the inequality (22) yields:

$$
\mu(t)+c_{1} v \int_{-\infty}^{t}\left(|\sigma|^{2}+\lambda \mu\right) \mathrm{d} \tau \leqslant c_{2} \int_{-\infty}^{t}\left(\frac{c_{0}}{v} \lambda|\xi|^{2}+\frac{\Gamma_{n} \lambda}{|u|^{2}}\right) \mathrm{d} \tau
$$


where

$$
c_{1}(t)=\inf _{\tau \leqslant t} \mathrm{e}^{\tau} \int_{-\infty}^{\tau} \beta
$$

and

$$
c_{2}(t)=\sup _{\tau \leqslant t} \mathrm{e}_{-\infty}^{\tau} \beta
$$

Observe that the relation (15) from Lemma 1 implies that

$$
\int_{-\infty}^{t} \frac{\Gamma_{n} \lambda}{|u|^{2}} \mathrm{~d} \tau \leqslant \frac{\Gamma_{n}}{v|u(t)|^{2}}
$$

Using this, together with (16), in the inequality (23), we obtain

$$
\mu(t)+c_{1} v \int_{-\infty}^{t}\left(|\sigma|^{2}+\lambda \mu\right) d \tau \leqslant \frac{c_{2} c_{0}}{v^{2}}\left(\frac{1}{2}\left(\lambda_{n}^{2}-\lambda^{2}(t)\right)+\frac{|g|^{2}}{v^{2}|u(t)|^{2}}\right)+\frac{c_{2} \Gamma_{n}}{v|u(t)|^{2}} .
$$

Observe that from (24) we can infer that $\mu(t)$ is bounded, while $|\sigma|^{2}$ and $\lambda \mu$ are integrable on $(-\infty, t]$, and thus

$$
c_{1} v \int_{-\infty}^{t} \frac{\left|A^{3 / 2} u\right|^{2}}{|u|^{4}} \mathrm{~d} \tau \leqslant c_{1} v \int_{-\infty}^{t} 3 \mu \lambda \mathrm{d} \tau+\frac{c_{2} c_{0}}{2 v^{2}}\left(\lambda_{n}^{2}-\lambda^{2}(t)\right)+\left(\frac{c_{0}|g|^{2}}{v^{3}}+\Gamma_{n}\right) \frac{c_{2}}{v|u(t)|^{2}} .
$$

Using (24) again to estimate $c_{1} v \int_{-\infty}^{t} 3 \mu \lambda \mathrm{d} \tau$, we obtain

$$
c_{1} v \int_{-\infty}^{t} \frac{\left|A^{3 / 2} u\right|^{2}}{|u|^{4}} \mathrm{~d} \tau \leqslant \frac{2 c_{2} c_{0}}{v^{2}}\left(\lambda_{n}^{2}-\lambda^{2}(t)\right)+\left(\frac{c_{0}|g|^{2}}{v^{3}}+\Gamma_{n}\right) \frac{4 c_{2}}{v|u(t)|^{2}}<\infty .
$$

Observe that from (15) we obtain that

$$
v \int_{-\infty}^{t} \frac{\lambda}{|u|} \mathrm{d} \tau \leqslant \frac{3}{2|u(t)|}
$$

Hence,

$$
c_{1}>\mathrm{e}^{-\int_{-\infty}^{0}(4|g| /|u|)} \geqslant \mathrm{e}^{-6|g| /\left(\nu \lambda_{1}|u(0)|\right)} \geqslant \mathrm{e}^{-3}
$$

and, since $|u| \geqslant \gamma_{0} \geqslant v$,

$$
c_{2} \leqslant \mathrm{e}^{\int_{-\infty}\left(v^{3} \lambda_{1} /|u|^{2}+4|g| /|u|\right)} \leqslant \mathrm{e}^{v^{2} /|u(t)|^{2}+6|g| /\left(\nu \lambda_{1}|u(t)|\right)} \leqslant \mathrm{e}^{1+3}=\mathrm{e}^{4} .
$$

Finally, if we define

$$
K_{1}\left(G_{0}, G_{1}\right):=\mathrm{e}^{4}\left(c_{0} G_{0}+G_{1}\right),
$$

and use (24) and (25) we will obtain the desired estimates from the proposition.

The following proposition allows us to deduce the boundedness of higher order quotients based on the boundedness on the lower ones. 
Proposition 2. Let $g \in D\left(A^{\theta+1 / 2}\right)$ with $\theta=k / 2$ and $k \in \mathbb{N}$. Suppose that for every $u(t) \in \mathcal{M}_{n},\left|u\left(t_{0}\right)\right| \geqslant \gamma_{0}$ we have

$$
\int_{-\infty}^{t_{0}} \frac{\left|A^{\theta} u(t)\right|^{2}}{|u(t)|^{m}} \mathrm{~d} t<\frac{C_{\theta, m}\left(G_{\theta-1 / 2}\right)}{\nu^{m-1}}{\overline{\lambda_{n}}}^{k-1},
$$

where $C_{\theta, m}(\cdot)$ is a positive increasing function. Then there exist positive increasing functions $C_{\theta, m}^{\prime}(\cdot), K_{\theta, m}(\cdot)$, such that

$$
\frac{\left|A^{\theta} u\left(t_{0}\right)\right|^{2}}{\left|u\left(t_{0}\right)\right|^{m+2}}+v \int_{-\infty}^{t_{0}} \frac{\left|A^{\theta+1 / 2} u(t)\right|^{2}}{|u(t)|^{(m+2)}} \mathrm{d} t<\frac{C_{\theta, m}^{\prime}\left(G_{\theta}\right)}{v^{m}}{\overline{\lambda_{n}}}^{k}
$$

and

$$
\frac{\left|A^{\theta+1 / 2} u(t)\right|^{2}}{|u(t)|^{(m+4)}} \leqslant \frac{K_{\theta, m}\left(G_{\theta+1 / 2}\right)}{v^{m+2}} \bar{\lambda}_{n}^{k+1} .
$$

Moreover,

$$
\lim _{t \rightarrow-\infty} \frac{\left|A^{\theta+1 / 2} u(t)\right|^{2}}{|u(t)|^{(m+4)}}=\lim _{t \rightarrow-\infty} \frac{\left|A^{\theta} u(t)\right|^{2}}{|u(t)|^{(m+2)}}=0 .
$$

Proof. Using (2) we get the following equation for the Galerkin approximations $u^{N}$ (cf. [2] for the facts about the Galerkin approximations for the NSE)

$$
\begin{aligned}
\frac{1}{2} \frac{\mathrm{d}}{\mathrm{d} t} \mu_{\theta, m+2}^{N}= & \frac{-v\left|A^{\theta+1 / 2} u^{N}\right|^{2}+\left(g, A^{2 \theta} u^{N}\right)-b\left(u^{N}, u^{N}, A^{2 \theta} u^{N}\right)}{\left|u^{N}\right|^{(m+2)}} \\
& +\frac{m+2}{2} \mu_{\theta, m+2}^{N} \frac{v\left|A^{1 / 2} u^{N}\right|^{2}-\left(g, u^{N}\right)}{\left|u^{N}\right|^{2}} .
\end{aligned}
$$

Applying Theorem 3 from the Appendix as well as the Cauchy-Schwarz inequality, we get

$$
\begin{aligned}
\frac{1}{2} \frac{\mathrm{d}}{\mathrm{d} t} \mu_{\theta, m+2}^{N} \leqslant & -v \mu_{\theta+1 / 2, m+2}^{N}+\frac{\left|A^{\theta} g\right|}{\left|u^{N}\right|^{(m+2) / 2}} \mu_{\theta, m+2}^{N}+c_{0} c_{2 \theta} \frac{\left|A^{\theta+1 / 2} u^{N}\right|\left|A^{\theta} u^{N}\right|\left|A^{1 / 2} u^{N}\right|}{\left|u^{N}\right|^{(m+2)}} \\
& +v \frac{m+2}{2} \lambda \mu_{\theta, m+2}^{N}+\frac{m+2}{2} \frac{|g|}{\left|u^{N}\right|} \mu_{\theta, m+2}^{N}
\end{aligned}
$$

(here $c_{2 \theta}=6\left([\theta]+(2 \theta-[\theta]) 2^{2 \theta-2}\right)$ is the constant from Theorem 3). Now, using the Jensen inequality, we obtain

$$
\begin{aligned}
\frac{1}{2} \frac{\mathrm{d}}{\mathrm{d} t} \mu_{\theta, m+2}^{N} \leqslant & -\frac{v}{2} \mu_{\theta+1 / 2, m+2}^{N}+\frac{1}{2 v \lambda_{1}} \frac{\left|A^{\theta} g\right|^{2}}{\left|u^{N}\right|^{(m+2)}}+\frac{v \lambda_{1}}{2} \mu_{\theta, m+2}^{N}+\frac{c_{0}^{2} c_{2 \theta}^{2}}{2 v} \lambda^{N} \mu_{\theta, m}^{N} \\
& +v \frac{m+2}{2} \lambda^{N} \mu_{\theta, m+2}^{N}+\frac{m+2}{2} \frac{|g|}{\left|u^{N}\right|} \mu_{\theta, m+2}^{N} .
\end{aligned}
$$

Hence,

$$
\begin{aligned}
& \frac{\mathrm{d}}{\mathrm{d} t} \mu_{\theta, m+2}^{N}+v \mu_{\theta+1 / 2, m+2}^{N} \\
& \quad \leqslant \frac{\left|A^{\theta} g\right|^{2}}{v \lambda_{1}\left|u^{N}\right|^{(m+2)}}+\left(\frac{v \lambda_{1}}{\left|u^{N}\right|^{2}}+\frac{c_{0}^{2} c_{2 \theta}^{2}}{v} \lambda^{N}+\frac{v(m+2) \lambda^{N}}{\left|u^{N}\right|^{2}}+\frac{(m+2)|g|}{\left|u^{N}\right|^{3}}\right) \mu_{\theta, m}^{N} .
\end{aligned}
$$

Since $g \in D\left(A^{\theta+1 / 2}\right)$, we can integrate from $t$ to $t_{0}\left(t<t_{0}\right)$ and pass to the limit $N \rightarrow \infty$. Taking into the account that $\lambda(t) \leqslant \bar{\lambda}_{n}$ we get 


$$
\begin{aligned}
& \mu_{\theta, m+2}\left(t_{0}\right)+v \int_{t}^{t_{0}} \mu_{\theta+1 / 2, m+2} \mathrm{~d} \tau \leqslant \mu_{\theta, m+2}(t)+\frac{\left|A^{\theta} g\right|^{2}}{v \lambda_{1}} \int_{t}^{t_{0}} \frac{\mathrm{d} \tau}{|u|^{m+2}} \\
& +\left[\frac{c_{0}^{2} c_{2 \theta}^{2}}{v} \bar{\lambda}_{n}+\frac{1}{\left|u\left(t_{0}\right)\right|^{2}}\left(v \lambda_{1}+v(m+2) \bar{\lambda}_{n}+\frac{(m+2)|g|}{\left|u\left(t_{0}\right)\right|}\right)\right] \int_{t}^{t_{0}} \mu_{\theta, m} \mathrm{~d} \tau .
\end{aligned}
$$

Since

$$
\int_{-\infty}^{t_{0}} \mu_{\theta, m} \mathrm{~d} \tau<\frac{C_{\theta, m}\left(G_{\theta-1 / 2}\right)}{\nu^{m-1}} \bar{\lambda}_{n}{ }^{k-1},
$$

there exists a sequence $t_{l} \rightarrow-\infty$ such that

$$
\lim _{l \rightarrow \infty} \mu_{\theta, m}\left(t_{l}\right)=0 \quad\left(=\lim _{l \rightarrow \infty} \mu_{\theta, m+2}\left(t_{l}\right)\right) .
$$

Thus, by letting $t=t_{l} \rightarrow-\infty$, we get

$$
\begin{aligned}
& \mu_{\theta, m+2}\left(t_{0}\right)+v \int_{-\infty}^{t_{0}} \mu_{\theta+1 / 2, m+2} \mathrm{~d} \tau \\
& \leqslant \frac{\left|A^{\theta} g\right|^{2}}{v \lambda_{1}} \int_{-\infty}^{t_{0}} \frac{\mathrm{d} \tau}{|u|^{m+2}}+\left[\frac{c_{0}^{2} c_{2 \theta}^{2}}{v} \bar{\lambda}_{n}+\frac{1}{\left|u\left(t_{0}\right)\right|^{2}}\left(v \lambda_{1}+v(m+2) \bar{\lambda}_{n}+\frac{(m+2)|g|}{\left|u\left(t_{0}\right)\right|}\right)\right] \int_{-\infty}^{t_{0}} \mu_{\theta, m} \mathrm{~d} \tau .
\end{aligned}
$$

Hence,

$$
\lim _{t \rightarrow-\infty} \mu_{\theta, m+2}(t)=0 .
$$

Moreover, since according to Lemma 1,

$$
v \int_{-\infty}^{t_{0}} \frac{\lambda}{|u|^{m+2}} \mathrm{~d} \tau \leqslant \frac{2}{m+2} \frac{1}{\left|u\left(t_{0}\right)\right|^{m+2}},
$$

we obtain

$$
\begin{aligned}
& \mu_{\theta, m+2}\left(t_{0}\right)+\int_{-\infty}^{t_{0}} \mu_{\theta+1 / 2, m+2} \mathrm{~d} \tau \\
& \leqslant \frac{\left|A^{\theta} g\right|^{2}}{v^{2} \lambda_{1}^{2}} \frac{2}{m+2} \frac{1}{\gamma_{0}^{m+2}}+\left[\frac{c_{0}^{2} c_{2 \theta}^{2}}{v} \bar{\lambda}_{n}+\frac{1}{\gamma_{0}^{2}}\left(v \lambda_{1}+v(m+2) \bar{\lambda}_{n}+\frac{(m+2)|g|}{\gamma_{0}}\right)\right] \frac{C_{\theta, m}\left(G_{\theta-1 / 2}\right)}{v^{m-1}} \bar{\lambda}_{n}^{k-1} .
\end{aligned}
$$

Observe that by the Poincaré inequality, $G_{\theta}>G_{\theta-1 / 2}$. Thus $C_{\theta, m}\left(G_{\theta-1 / 2}\right) \leqslant C_{\theta, m}\left(G_{\theta}\right)$. Using this fact, together with the definition of $\gamma_{0}$, we can define the positive increasing functions $C_{\theta, m}^{\prime}\left(G_{\theta}\right)$ from the statement of the proposition as follows:

$$
\begin{aligned}
& \frac{2\left|A^{\theta} g\right|^{2} v^{m}}{(m+2) v^{2} \lambda_{1}^{2+k} \gamma_{0}^{m+2}}+\left[c_{0}^{2} c_{2 \theta}^{2}+\frac{v^{2}}{\gamma_{0}^{2}}\left(1+(m+2)\left(1+\frac{|g|}{v \lambda_{1} \gamma_{0}}\right)\right)\right] C_{\theta, m}\left(G_{\theta-1 / 2}\right) \\
& =\frac{2}{m+2} G_{\theta}^{2}+\left(c_{0}^{2} c_{2 \theta}^{2}+\frac{3}{2} m+4\right) C_{\theta, m}\left(G_{\theta-1 / 2}\right) \\
& \leqslant \frac{2}{m+2} G_{\theta}^{2}+\left(c_{0}^{2} c_{2 \theta}^{2}+\frac{3}{2} m+4\right) C_{\theta, m}\left(G_{\theta}\right):=C_{\theta, m}^{\prime}\left(G_{\theta}\right) .
\end{aligned}
$$


On the other hand, again for the Galerkin approximations, we have

$$
\begin{aligned}
\frac{1}{2} \frac{\mathrm{d}}{\mathrm{d} t} \mu_{\theta+1 / 2, m+4}^{N}= & \frac{-v\left|A^{\theta+1} u^{N}\right|^{2}+\left(g, A^{2 \theta+1} u^{N}\right)-b\left(u^{N}, u^{N}, A^{2 \theta+1} u^{N}\right)}{\left|u^{N}\right|^{(m+4)}} \\
& +\frac{m+4}{2} \mu_{\theta+1 / 2, m+4}^{N} \frac{v\left|A^{1 / 2} u^{N}\right|^{2}-\left(g, u^{N}\right)}{\left|u^{N}\right|^{2}},
\end{aligned}
$$

and similarly to what was done above we get

$$
\begin{gathered}
\mu_{\theta+1 / 2, m+4}\left(t_{0}\right)+v \int_{t}^{t_{0}} \mu_{\theta+1, m+4} \mathrm{~d} \tau \leqslant \mu_{\theta+1 / 2, m+4}(t)+\frac{\left|A^{\theta+1 / 2} g\right|^{2}}{\nu \lambda_{1}} \int_{t}^{t_{0}} \frac{\mathrm{d} \tau}{|u|^{(m+4)}} \\
+\left[\frac{c_{0}^{2} c_{2 \theta+1}^{2}}{v} \bar{\lambda}_{n}+\frac{1}{\left|u\left(t_{0}\right)\right|^{2}}\left(v \lambda_{1}+(m+4)\left(v \bar{\lambda}_{n}+\frac{|g|}{\left|u\left(t_{0}\right)\right|}\right)\right)\right] \int_{t}^{t_{0}} \mu_{\theta+1 / 2, m+2} \mathrm{~d} \tau
\end{gathered}
$$

(here again $c_{2 \theta+1}$ is the constant from Theorem 3). By the same argument as in the previous case, when $t \rightarrow-\infty$ we obtain

$$
\begin{aligned}
& \mu_{\theta+1 / 2, m+4}\left(t_{0}\right)+v \int_{-\infty}^{t_{0}} \mu_{\theta+1, m+4} \mathrm{~d} \tau \leqslant \frac{\left|A^{\theta+1 / 2} g\right|^{2}}{\nu \lambda_{1}} \int_{-\infty}^{t_{0}} \frac{\mathrm{d} \tau}{|u|^{(m+4)}} \\
& +\left[\frac{c_{0}^{2} c_{2 \theta+1}^{2}}{v} \bar{\lambda}_{n}+\frac{1}{\left|u\left(t_{0}\right)\right|^{2}}\left(v \lambda_{1}+(m+4)\left(v \bar{\lambda}_{n}+\frac{|g|}{\left|u\left(t_{0}\right)\right|}\right)\right)\right] \int_{-\infty}^{t_{0}} \mu_{\theta+1 / 2, m+2} \mathrm{~d} \tau .
\end{aligned}
$$

Thus

$$
\lim _{t \rightarrow-\infty} \mu_{\theta+1 / 2, m+4}(t)=0
$$

and

$$
\mu_{\theta+1 / 2, m+4}(t) \leqslant \frac{K_{\theta, m}}{v^{m+2}} \bar{\lambda}_{n}^{k+1},
$$

where

$$
K_{\theta, m}\left(G_{\theta+1 / 2}\right):=\frac{2}{m+4} G_{\theta+1 / 2}^{2}+\left(c_{0}^{2} c_{2 \theta+1}^{2}+\frac{3}{2} m+7\right) C_{\theta, m}^{\prime}\left(G_{\theta+1 / 2}\right) .
$$

Observe that $K_{\theta, m}$ satisfies conditions from the proposition, since

$$
\begin{gathered}
\frac{2\left|A^{\theta+1 / 2} g\right|^{2} v^{m+4}}{(m+4) v^{4} \lambda_{1}^{k+3} \gamma_{0}^{m+4}}+\left[c_{0}^{2} c_{2 \theta+1}^{2}+\frac{v^{2}}{\gamma_{0}^{2}}\left(1+(m+4)\left(1+\frac{|g|}{\lambda_{1} v \gamma_{0}}\right)\right)\right] C_{\theta, m}^{\prime}\left(G_{\theta}\right) \\
=\frac{2}{m+4} G_{\theta+1 / 2}^{2}+\left(c_{0}^{2} c_{2 \theta+1}^{2}+\frac{3}{2} m+7\right) C_{\theta, m}^{\prime}\left(G_{\theta}\right) \leqslant K_{\theta, m}\left(G_{\theta+1 / 2}\right)
\end{gathered}
$$

Proof of the main theorem. We will prove Theorem 2 by induction on $k=2 \theta$.

When $k=1$ the theorem holds (cf. (9)).

When $k=2$ the theorem is valid via Proposition 1. Observe that this proposition allows us to choose, for example,

$$
M_{2}\left(G_{1}\right)=\left(\left(c_{0}+1\right) G_{1}+\frac{15}{4}\right) \mathrm{e}^{4} .
$$


Moreover, Proposition 1 gives us that

$$
N_{3 / 2}=4 \mathrm{e}^{3} M_{2} \text {. }
$$

Thus, applying Proposition 2, we conclude that the theorem holds when $k=3$.

Suppose now that the theorem is true for some integer $k \geqslant 3$. Then there exists a positive increasing function $N_{\theta}(\cdot)$, such that

$$
\int_{-\infty}^{t_{0}} \frac{\left|A^{\theta} u\right|^{2}}{|u|^{4 \theta-2}} \mathrm{~d} \tau<\frac{N_{\theta}\left(G_{\theta-1 / 2}\right)}{v^{4 \theta-3}} \bar{\lambda}_{n}^{2 \theta-1},
$$

where $\theta=k / 2$. But according to Proposition 2, if $g \in D\left(A^{\theta+1 / 2}\right)$, then there exist positive increasing functions $M_{\theta+1 / 2}(\cdot)$ and $N_{\theta+1 / 2}(\cdot)$ such that

$$
\begin{gathered}
\int_{-\infty}^{t_{0}} \frac{\left|A^{\theta+1 / 2} u(t)\right|^{2}}{|u(t)|^{4 \theta}} \mathrm{d} t<\frac{N_{\theta+1 / 2}\left(G_{\theta}\right)}{v^{4 \theta-1}} \bar{\lambda}_{n}^{2 \theta}, \\
\frac{\left|A^{\theta+1 / 2} u_{0}\right|^{2}}{\left|u_{0}\right|^{4 \theta+2}} \leqslant \frac{M_{\theta+1 / 2}\left(G_{\theta+1 / 2}\right)}{v^{4 \theta}} \bar{\lambda}_{n}^{2 \theta+1},
\end{gathered}
$$

and

$$
\lim _{t \rightarrow-\infty} \frac{\left|A^{\theta+1 / 2} u(t)\right|^{2}}{|u(t)|^{4 \theta+2}}=0,
$$

which shows that the theorem is true for the integer $k+1$, and by induction, the proof is complete.

\section{Acknowledgements}

The author would like to thank Professor Ciprian Foias for helpful discussions, suggestions, and comments.

\section{Appendix. Estimate for the nonlinear term (cf. [5])}

Lemma 2. For each $n \in \mathbb{N}(n \geqslant 2)$ and every $u \in D\left(A^{n}\right)$ :

$$
b\left(u, u, A^{n} u\right)=-\sum_{h=1}^{n-1} b\left(A^{h} u, u, A^{n-h} u\right) .
$$

Proof. Observe first that

$$
A(B(u, v)+B(v, u))=B(u, A v)+B(v, A u)-B(A u, v)-B(A v, u) .
$$

Thus, if $n$ is odd, then

$$
\begin{aligned}
A \sum_{h=0}^{n} B\left(A^{h} u, A^{n-h} u\right) & =\sum_{h=0}^{(n+1) / 2} A\left(B\left(A^{h} u, A^{n-h} u\right)+B\left(A^{n-h} u, A^{h} u\right)\right) \\
& =\sum_{h=0}^{(n+1) / 2} B\left(A^{h} u, A^{n-h+1} u\right)+\sum_{h=0}^{(n+1) / 2} B\left(A^{n-h} u, A^{h+1} u\right)
\end{aligned}
$$




$$
\begin{aligned}
& -\sum_{h=0}^{(n+1) / 2} B\left(A^{h+1} u, A^{n-h} u\right)-\sum_{h=0}^{(n+1) / 2} B\left(A^{n-h+1} u, A^{h} u\right) \\
= & B\left(u, A^{n+1} u\right)-B\left(A^{n+1} u, u\right) .
\end{aligned}
$$

Consequently, in this case,

$$
\begin{aligned}
\sum_{h=0}^{n} b\left(A^{h} u, u, A^{n-h} u\right) & =-\sum_{h=0}^{n} b\left(A^{h} u, A^{n-h} u, u\right)=-\left(A \sum_{h=0}^{n} B\left(A^{h} u, A^{n-h} u\right), A^{-1} u\right) \\
& =-b\left(u, A^{n+1}, A^{-1} u\right)+b\left(A^{n+1} u, u, A^{-1} u\right) \\
& =b\left(u, A^{-1} u, A^{n+1} u\right)-b\left(A^{n+1} u, A^{-1} u, u\right)=0,
\end{aligned}
$$

since

$$
b(A v, v, w)=b(w, v, A v)
$$

for every $v \in D(A)$ and $w \in H$.

If $n$ is even, we have

$$
\begin{aligned}
A \sum_{h=0}^{n} B\left(A^{h} u, A^{n-h} u\right) & =A B\left(A^{n} u, u\right)+\sum_{h=0}^{n / 2} A\left(B\left(A^{h} u, A^{n-h} u\right)+B\left(A^{n-h} u, A^{h} u\right)\right) \\
& =A B\left(A^{n} u, u\right)+B\left(u, A^{n} u\right)-B\left(A^{n} u, u\right) .
\end{aligned}
$$

Thus,

$$
\begin{aligned}
\sum_{h=0}^{n} b\left(A^{h} u, u, A^{n-h} u\right) & =-\sum_{h=0}^{n} b\left(A^{h} u, A^{n-h} u, u\right)=-\left(A \sum_{h=0}^{n} B\left(A^{h} u, A^{n-h} u\right), A^{-1} u\right) \\
& =-\left(A B\left(A^{n} u, u\right), A^{-1} u\right)-b\left(u, A^{n+1} u, A^{-1} u\right)+b\left(A^{n+1} u, u, A^{-1} u\right) \\
& =b\left(A^{n} u, u, u\right)+0=0 .
\end{aligned}
$$

Consequently the identity from the lemma holds for all $n$.

Theorem 3. For each $n \in \mathbb{N}(n \geqslant 2)$ and every $u \in D\left(A^{n}\right)$ :

$$
\left|b\left(u, u, A^{n} u\right)\right| \leqslant c_{0} c_{n}\left|A^{n / 2} u\right|\left|A^{(n+1) / 2} u\right|\left|A^{1 / 2} u\right|,
$$

where $c_{n}:=6\left([n / 2]+(n-[n / 2]) 2^{n-2}\right)$.

Proof. Observe that going to the Fourier coefficients:

$$
|b(u, v, w)|=\left|\sum_{j+k+l=0 \in \mathbb{Z}^{2}}\left(a_{k} \cdot j\right)\left(b_{j} \cdot c_{l}\right)\right| \leqslant \sum_{j+k+l=0 \in \mathbb{Z}^{2}}\left|a_{k}\right||j|\left|b_{j}\right|\left|c_{l}\right|:=\tilde{b}(u, v, w),
$$

where $u(x)=\sum_{k \in \mathbb{Z}^{2}} a_{k} \mathrm{e}^{(2 \pi i / L)(k \cdot x)}, v(x)=\sum_{j \in \mathbb{Z}^{2}} b_{j} \mathrm{e}^{(2 \pi i / L)(j \cdot x)}$, and $w(x)=\sum_{l \in \mathbb{Z}^{2}} c_{l} \mathrm{e}^{(2 \pi i / L)(l \cdot x)}$, with $u, w \in H, v \in V$.

Using the previous lemma we get

$$
\left|b\left(u, u, A^{n} u\right)\right| \leqslant \sum_{h=1}^{n-1}\left|b\left(A^{h} u, u, A^{n-h} u\right)\right| \leqslant \sum_{h=1}^{n-1} \tilde{b}\left(A^{h} u, u, A^{n-h} u\right) .
$$

Observe that 


$$
\begin{aligned}
\sum_{h=1}^{n-1} \tilde{b}\left(A^{h} u, u, A^{n-h} u\right) & =\sum_{h=1}^{n-1} \sum_{j+k+l=0 \in \mathbb{Z}^{2}}|k|^{2 h}\left|a_{k}\right||j|\left|a_{j}\right|\left|a_{l}\right||l|^{2(n-h)} \\
& \leqslant A+B+C
\end{aligned}
$$

where

$$
\begin{aligned}
& A:=\sum_{h=1}^{n-1} \sum_{j+k+l=0 \in \mathbb{Z}^{2},|k| \leqslant \min \{|j|,|l|\}}|k|^{2 h}\left|a_{k}\right||j|\left|a_{j}\right|\left|a_{l}\right||l|^{2(n-h),}, \\
& B:=\sum_{h=1}^{n-1} \sum_{j+k+l=0 \in \mathbb{Z}^{2},|j| \leqslant \min \{|k|,|l|\}}|k|^{2 h}\left|a_{k}\right||j|\left|a_{j}\right|\left|a_{l}\right||l|^{2(n-h),},
\end{aligned}
$$

and

$$
C:=\sum_{h=1}^{n-1} \sum_{j+k+l=0 \in \mathbb{Z}^{2},|l| \leqslant \min \{|j|,|k|\}}|k|^{2 h}\left|a_{k}\right||j|\left|a_{j}\right|\left|a_{l}\right||l|^{2(n-h)} .
$$

Because of the symmetry we have that $A=C$. Also,

$$
B \geqslant \sum_{h=1}^{n-1} \sum_{j+k+l=0 \in \mathbb{Z}^{2},|k| \leqslant \min \{|j|,|l|\}}|k|^{2 h}\left|a_{k}\right||j|^{2(n-h)}\left|a_{j}\right|\left|a_{l}\right||l|=C(=A),
$$

and thus we get

$$
\begin{aligned}
\left|b\left(u, u, A^{n} u\right)\right| \leqslant 3 B \leqslant & 3 \sum_{h=1}^{n-1} 2 \sum_{j+k+l=0 \in \mathbb{Z}^{2},|j| \leqslant|l| \leqslant|k|}|k|^{2 h}\left|a_{k}\right||j|\left|a_{j}\right|\left|a_{l}\right||l|^{2(n-h)} \\
= & 6 \sum_{h=1}^{[n / 2]} \sum_{j+k+l=0 \in \mathbb{Z}^{2},|j| \leqslant|l| \leqslant|k|}|k|^{2 h}\left|a_{k}\right||j|\left|a_{j}\right|\left|a_{l}\right||l|^{2(n-h)} \\
& +6 \sum_{h=[n / 2]+1} \sum_{j+k+l=0 \in \mathbb{Z}^{2},|j| \leqslant|l| \leqslant|k|}|k|^{2 h}\left|a_{k}\right||j|\left|a_{j}\right|\left|a_{l}\right||l|^{2(n-h)} \\
\leqslant & 6 \sum_{h=1}^{[n / 2]} \sum_{j+k+l=0 \in \mathbb{Z}^{2},|j| \leqslant|l| \leqslant|k|}|k|^{n}\left|a_{k}\right||j|\left|a_{j}\right|\left|a_{l}\right||l|^{n} \\
& +6 \sum_{h=[n / 2]+1}^{n-1} \sum_{j+k+l=0 \in \mathbb{Z}^{2},|j| \leqslant|l| \leqslant|k|}|k|^{2 h}\left|a_{k}\right||j|\left|a_{j}\right|\left|a_{l}\right||l|^{2(n-h) .} .
\end{aligned}
$$

Observe that in the previous sums, $|k|=|j+l| \leqslant|j|+|l| \leqslant 2|l|$. Thus,

$$
\begin{aligned}
& 6 \sum_{h=[n / 2]+1}^{n-1} \sum_{j+k+l=0 \in \mathbb{Z}^{2},|j| \leqslant|l| \leqslant|k|}|k|^{2 h}\left|a_{k}\right||j|\left|a_{j}\right|\left|a_{l}\right||l|^{2(n-h)} \\
& \leqslant 6 \sum_{h=[n / 2]+1}^{n-1} \sum_{j+k+l=0 \in \mathbb{Z}^{2},|j| \leqslant|l| \leqslant|k|}|k|^{n}(2|l|)^{2 h-n}\left|a_{k}\right||j|\left|a_{j}\right|\left|a_{l}\right||l|^{2(n-h)}
\end{aligned}
$$




$$
\leqslant 6(n-[n / 2]) 2^{n-2} \sum_{j+k+l=0 \in \mathbb{Z}^{2},|j| \leqslant|l| \leqslant|k|}|k|^{n}\left|a_{k}\right||j|\left|a_{j}\right|\left|a_{l}\right||l|^{n} .
$$

Also,

$$
\begin{aligned}
& 6 \sum_{h=1}^{[n / 2]} \sum_{j+k+l=0 \in \mathbb{Z}^{2},|j| \leqslant|l| \leqslant|k|}|k|^{n}\left|a_{k}\right||j|\left|a_{j}\right|\left|a_{l}\right||l|^{n} \\
& \quad \leqslant 6([n / 2]) \sum_{j+k+l=0 \in \mathbb{Z}^{2},|j| \leqslant|l| \leqslant|k|}|k|^{n}\left|a_{k}\right||j|\left|a_{j}\right|\left|a_{l}\right||l|^{n} .
\end{aligned}
$$

Consequently,

$$
\left|b\left(u, u, A^{n} u\right)\right| \leqslant 6\left([n / 2]+(n-[n / 2]) 2^{n-2}\right) \sum_{j+k+l=0 \in \mathbb{Z}^{2},|j| \leqslant|l| \leqslant|k|}|k|^{n}\left|a_{k}\right||j|\left|a_{j}\right|\left|a_{l}\right||l|^{n} .
$$

Let $c_{n}=6\left([n / 2]+(n-[n / 2]) 2^{n-2}\right)$. From the above we conclude that

$$
\left|b\left(u, u, A^{n} u\right)\right| \leqslant c_{n} \tilde{b}\left(A^{n / 2} u, u, A^{n / 2} u\right)=c_{n} \int_{\Omega} \phi(x) \psi(x) \zeta(x) \mathrm{d} x,
$$

where we denote $\phi(x)=\sum_{k} \mathrm{e}^{(2 \pi \mathrm{i} / L) k \cdot x}\left|a_{k}\right||k|^{n}, \psi(x)=\sum_{j} \mathrm{e}^{(2 \pi \mathrm{i} / L) j \cdot x}\left|a_{j}\right||j|$, and $\zeta(x)=\sum_{l} \mathrm{e}^{(2 \pi \mathrm{i} / L) l \cdot x}\left|a_{l}\right||l|^{n}$. Applying Schwartz inequality we get

$$
\left|b\left(u, u, A^{n} u\right)\right| \leqslant c_{n}|\phi|_{L^{4}}|\psi|_{L^{2}}|\zeta|_{L^{4}} .
$$

Now apply Ladyzhenskaya inequality

$$
|w|_{L^{4}}^{2} \leqslant c_{0}|w|_{H^{1}}|w|_{L^{2}}
$$

to estimate $|\phi|_{L^{4}}$ and $|\zeta|_{L^{4}}$ and obtain

$$
\left|b\left(u, u, A^{n} u\right)\right| \leqslant c_{0} c_{n}\left|A^{n / 2} u\right|\left|A^{(n+1) / 2} u\right|\left|A^{1 / 2} u\right| .
$$

\section{References}

[1] C. Bardos, L. Tartar, Sur l'unicité rétrograde des équations parabolique et quelques questiones voisines, Arch. Rational Mech. 50 (1973) 10-25.

[2] P. Constantin, C. Foias, Navier-Stokes Equations, Chicago Lectures in Math., University of Chicago Press, Chicago, 1988.

[3] P. Constantin, C. Foias, I. Kukavica, A. Majda, Dirichlet quotients and 2-d periodic Navier-Stokes equations, J. Math. Pures Appl. 76 (1997) 125-153.

[4] R. Dascaliuc, On the backward-time behavior of Burgers' original model for turbulence, Nonlinearity 16 (6) (2003) $1945-1965$.

[5] C. Foias, B. Nicolaenko, Some estimates on the nonlinear term of the Navier-Stokes equation, Preprint, 2003.

[6] I. Kukavica, On the behavior of the solutions of the Kuramoto-Sivashinsky equations for negative time, J. Math. Anal. Appl. 166 (1992) 601-606.

[7] I. Kukavica, M. Malcok, Backward behavior of solutions of Kuramoto-Sivashinsky equation, 2003, submitted for publication.

[8] I. Richards, On the gaps between numbers which are sums of two squares, Adv. in Math. 46 (1982) 1-2.

[9] R. Temam, Navier-Stokes Equations and Nonlinear Functional Analysis, SIAM, Philadelphia, 1983.

[10] J. Vukadinovic, On the backwards behavior of the solutions of the 2D periodic viscous Kamassa-Holm equations, J. Dynam. Differential Equations 14 (2) (2002).

[11] J. Vukadinovic, On the backwards behavior of the solutions of the Kelvin-filtered 2D periodic Navier-Stokes equations, Ph.D. Thesis, Indiana University, 2002. 